\title{
Excellence in Practice through a Socio-Technical, Open Systems Approach to Process Analysis and Design
}

\author{
Peter M. Bednar, School of Computing, University of Portsmouth, UK \\ Dr Christine Welch, University of Portsmouth Business School, UK \\ Christopher Milner, University of Portsmouth Business School, UK
}

\begin{abstract}
Nowadays, organizations pursue their aims in a context of distributed collaboration, creating a need not only for supporting work systems, but for a human-centred focus in which individual and group sense-making and learning are supported by appropriate toolsets. We argue that development of such toolsets requires an open systems approach. This paper discusses examples of such approaches, including non-competitive benchmarking (NCB), as a vehicle for knowledge transfer, leading to process improvement and potential for enhanced organizational performance. The paper goes on to discuss tools and techniques that may be used to support desire to reflect upon 'best practice' in socio-technical design, without losing contextual relevance in design. We use these examples to explore ways in which engaged actors may be supported to create and share their contextually-dependent tacit knowledge. The foundation of open systems approaches is discussed, showing how sociotechnical approaches continue to have relevance today.
\end{abstract}

Key words: Open systems approaches; socio-technical systems; non-competitive benchmarking; systems analysis practice; work design.

\section{Introduction}

Business managers are often heard to express a view that 'People are the company's greatest asset'. However, this view is not always translated into effective practice. We believe that a holistic, socio-cultural approach to organizational change, recognizing the contextuallydependent nature of work is clearly the best strategy to pursue. In order to empower people to contribute their contextually-relevant 'know-how', both as individuals and in communities of practice, two prerequisites can be identified. The first is an open culture of 'mindfulness' in which people feel supported to express their views, even where these conflict with accepted ideas (Weick and Sutcliffe, 2007, p.23); the second is a foundation for creativity in the form of useful and usable methodologies for inquiry and design of future practice (Bednar and Welch, 2006).

Any technical system developed to support human activities can only be regarded as worthwhile if it is experienced by the engaged actors as useful to them in their work roles (Bednar and Welch, 2009); and in order for technologies (including Information \& 
Communication Technologies) to be 'useful' they must play an instrumental role within an organizational setting. The purpose of technology implementation is therefore to bring about a change in organizational behavior. However, an organization can be viewed as a dynamic, open system that is created and recreated continuously over time by interactions among the human individuals who are its members (Mumford, 2006; Bednar, 2007). Change of behaviour is not determined by technologies; it requires purposeful engagement by the actors concerned. To illustrate this point, we will explore a case example: how Great Ormond Street Hospital for Children (GOSH) brought about needful change in their system for handover from one surgical team to another in infant care. This involved a novel endeavor in collaboration with the Ferrari and McClaren Formula 1 pit teams, and also with two aviator training captains. Through a process of non-competitive benchmarking, knowledge was shared among the teams and used by staff at GOSH to bring about beneficial change in their practice (Catchpole, et al, 2007).

In this paper, we discuss ways in which creative learning spirals may be established that support individuals to escape from entrapment in established routines and generate new protocols for enhanced performance through reflection (Bateson, 1972). In GOSH, staff in the infant coronary care field made use of non-competitive benchmarking with teams from fields as disparate from their normal experience as Formula 1 pit crews and pilot trainers from aviation. Their vision was to redesign their behavior and movements in the operational units concerned with infant heart surgery in order to reduce mortality rates.

In the past, many writers on organizations have referred to management as a practice of goal setting/seeking. It has been suggested that organizational culture is formed over time through shared goals and values (Schein, 1992). Such sharing, if possible, would require negotiation of differing perspectives held by individuals. Checkland (1999) suggests that "Consciousness makes man a meaning-endowed animal" (p.218). As such, it is always possible for each individual to select from a range of possible meanings. We consider each individual to have a multitude of competing worldviews, all of which change through time as a result of experience. Perceptions by about the same phenomenon different individuals within a group may overlap, but will vary from each other and will also change over time. For this reason, agreement on a single description of a 'real' human activity system will be elusive and consensus on its 'goals' impossible to achieve. Individuals coming together in organizational settings may develop views of a common good which they collectively pursue. However, it is unlikely that the collection of individuals interacting within that human activity system will all share the same perception of the nature of their system or of the nature of that 'common'.

We suggest that the purpose of 'knowledge' management within an organization is creation of vehicles to support people to create and share their individual, contextually-dependent understandings. 'Know-how' is embedded within individual people and groups scattered throughout an organization. Only by co-creating understandings of one another's individual perspectives can individuals begin to appreciate the similarities and differences in their views: their visions of the common. Individual perceptions of organizational culture, climate 
and collective image will co-exist within interacting groups, continually shifting over time. Perceptions of a collective organizational purpose suggest that individuals experience themselves to be engaged in common endeavors within self-defined boundaries (Bednar, 2007). These boundaries are also individually co-created and subject to continual change. The vision statements which organizations publish from time to time, intended to reflect an inward collective identity and outward collective image, even where these are genuinely derived from dialogue among organizational actors, can only represent a snapshot of a collective understanding which is perpetually shifting.

\section{Non-competitive Benchmarking as a Socio-technical Tool}

One example of an approach which could be helpful to individuals in creating and sharing both explicit and tacit knowledge is non-competitive benchmarking (NCB). In an economy where organisations are under increasing pressure to deliver operational efficiency, process improvement and competitive advantage, benchmarking can be an effective management tool. However, attempts to compare practice within the same field can lead to feelings of hostility and insecurity, leading to reluctance to share knowledge. NCB can help to overcome such feelings and help to promote sharing and learning among participants. The expression 'benchmarking' was first used in the Xerox Corporation during the 1980's and is essentially a method used to stimulating creativity in improvement practice. Slack et al (2010) define benchmarking as 'the process of learning from others'. Over the last 30 years it has become a staple tool, traditionally focused on 'best practice', which the American Productivity and Quality Centre suggest to consist of:

those practices that have been shown to produce superior results; selected by a systematic process; and judged as exemplary, good, or successfully demonstrated ... adapted to fit a particular organisation' Yasar and Zairi (2010, p.736).

This paper is focused in the value of external, non competitive benchmarking: the comparison between an operation in one organization and a comparable but dissimilar operation in another. We view this as an example of socio-technical practice, as it is a vehicle for knowledge creation and sharing. Organisations concerned do not compete in the same markets, but can collaborate as a virtual learning organisation, opening up novel ideas to processes, approaches and concerns. It is an opportunity for a company to observe, reflect upon and adopt and/or adapt the practices judged as 'best', with the aim to improve the performance of a given business process and so it is the processes that must have common ground, not the company or market they operate within. This process of creating productive learning spirals through observation and reflection-in-context contrasts with some other approaches to 'best practice' based in a naive belief that good practice can simply be transplanted, irrespective of context. 
Many well-known companies have used benchmarking as a tool for improvement, e.g. Avon, Exxon, Microsoft, Ford, and General Motors (Elmuti and Kathawala, 1997). Ohno reports how Toyota's new, just-in-time inventory system was developed following visits to a US Supermarket in 1956. Having observed their method for replenishing shelves, he was able to adapt what he had learned to inventory management (in Ahmed and Rafiq, 1998). Dorsch and Yasin (1998) comment on the early Xerox Logistics and Distribution example. Functional benchmarking through a non-competitor (L.L. Bean) led to successful implementation of greatly improved warehousing systems and more efficient picking processes. In a second example of a successful collaboration cited by Dorsch, these authors involved Nissan and a variety of partner organizations (e.g. Disney; McDonalds). Nissan were able to discover and adapt 'best' human resource practices relating to empowerment, teamwork, and focus on customer satisfaction.

\section{The Formula 1 and Infant Surgery}

Through an open environment for learning, observation and communication (sense-making), they were able to reflect upon excellence in practice in these very difficult fields in order to create and develop better processes to deliver care. Boxwell (1994) describes interactions of Great Ormond Street Hospital (GOSH) staff and Formula 1 motor racing teams as a form of collaborative benchmarking. Catchpole, et al (2007) report on this experiment as follows:

The pit-stop in Formula 1 motor racing was seen as a model example of how a multiprofessional team comes together as a single unit to effectively perform a complex task (change four tyres and fill with fuel) under huge time pressure (approx 7 s) with minimal error. ... This was targeted as an industry with analogies to the handover of patients from theatre to ICU where multiprofessional specialists (surgeons, anesthetist and ICU staff) reconfigure, as a single unit under time pressure, to safely transfer all equipment and information (2007, p.471).

GOSH wished to bring about improvement to its processes for handover of care in infant surgical procedures, and approached 'best in class' F1 teams to ask if they would be willing to share knowledge of their precision systems for supporting drivers. Use of benchmarking across dissimilar organisations may be very demanding, requiring a broad conceptualization of entire processes and careful understanding of procedures. However, it has been shown to be extremely effective.

The impetus for this exercise came from two doctors who were watching Formula 1 motor racing on TV while relaxing after lengthy surgeries at GOSH. Realising that F1 pit stops involved processes conceptually identical to those involved in changeover during infant surgery, they began to pay particular attention to them. GOSH treat approximately 500 paediatric cardiac cases every year. Co-ordination and quality of knowledge sharing in the handover of infants from the intensive care team to the theatre team before and after complex 
heart surgery has been found to be crucial to the success of the procedure, since patient's particular condition is unique (De Leval, et al, 2000). It became evident to the surgeons that there was a common interest in reducing error and improving quality between the two very different contexts. In both contexts, time was of the essence. They believed that precision in procedures achieved by a Formula 1 pit team could hold valuable lessons for improvements in handovers during operations. Catchpole, et.al (2007) and Sower et al, (2008) have written at length about this relationship. The pit stop in Formula 1 motor racing was seen as a model example of how a multi professional team collaborates to perform a complex task effectively, under huge time pressure, with minimal error. The aim of the benchmarking study was to observe and reflect upon this expertise and combine it with existing knowledge to develop simple, reliable, easily trainable handover protocols to improve safety and quality of patient care in the short but critical period after complex infant heart surgery.

GOSH surgical staff visited and observed the pit crew handover during a race, becoming increasingly interested in the way the team addressed possible failure 'What could go wrong?' 'What are we going to do if it does go wrong?' and 'How important is it if it does go wrong?' Benchmarking against the Ferrari team pushed the hospital to anticipate problems rather than waiting to deal with them as they arose. Observing Ferrari caused the hospital to view its own practice from a completely different perspective. Ferrari staff did not tell them what needed to be changed, or how. COSH staff performed similar observation/reflection cycles in relation to training systems for aviators - another situation in which time and error reduction were seen to be crucial. The hospital staff reflected upon their observations of good practice among pit crews, aviator trainers and others and adapted the lessons learned to fit their own situation.

The GOSH doctors noted the value in process mapping, process description, and trying to work out what individual tasks should be. Under the new handover process they devised, the anaesthetist was given overall responsibility for coordinating the team, acting in a way which was analogous to the pit crew 'lollypop man', and a dance choreographer was brought in to help the team position their movements so that they would not impede one another while carrying out their particular roles.

Adequate time and money allows motor racing teams to have rehearsal after rehearsal. In healthcare, where resources are scarce and teams are dealing with living people, a process is required that is simple, easy to learn, and does not depend upon a lot of rehearsal. Through the benchmarking process, GOSH doctors noted not only how fast, but also how quiet and disciplined the pit crews were, and designed new processes for the surgical teams intended to emulate this atmosphere of calm professionalism. Development of a new handover protocol reflected this influence very strongly, emphasizing such factors as teamwork, effective leadership, rhythm, standardised processes, anticipation, and communication [Catchpole, et al, pp 272 and 276]. Once in place, the protocol was polished further through reflection upon other contexts such as the work of aviator trainers. It was simple to understand and could be established within 15-30 min at staff induction. Results showed that the new handover 
procedure broke the link between technical and informational errors. Before the new protocol, approximately $30 \%$ of patient errors occurred in both equipment and information; afterward, only $10 \%$ of the patient errors occurred in both areas. While the new system was not perfect, GOSH identified significant improvement and adopted a policy of seeking further, continuous improvement in procedures.

The development of a simple, easily trainable handover process using expertise from other high-risk industries reduced errors and improved information transfer with no penalty in handover duration and reliable training overhead. GOSH believe this work may be extrapolated to handovers in other areas of medicine, and would encourage further attempts to evaluate this protocol systematically and to combine expertise from other industries with that of health care.

We have looked at non-competitive benchmarking as a collaborative, open systems approach to surfacing and sharing tacit knowledge. In the next section we will examine the usefulness of benchmarking techniques in process design.

\section{An open systems toolbox}

We suggest that an open systems approach which enables individuals to explore and share their contextually dependent understandings will be helpful in supporting exchange of tacit knowledge. Human systems create problem spaces that are ambiguous, uncertain and constantly changing. This is demonstrated particularly well in the case example of Great Ormond Street Hospital above. Tool-sets are therefore needed that can be used collaboratively, to explore inconsistent and ill-defined phenomena.

Design of business processes today is indivisible from Information Systems design. As Langefors (1995, p.53) pointed out, all management activity depends upon availability of data about the behaviour of the components of organizational systems. The organization and its processes are therefore synonymous with its Information System. The advent of modern Enterprise Resource Planning Systems (ERP) has lent widespread recognition to this (see, e.g. Moon, 2007). Many different interpretations of the term 'Information System' have been applied by researchers and practitioners, but two common and contrasting examples follow. In first order (what we can term IS1), a (socio-) technical system is most comprised of ICT's, with indications of their use by human actors. The boundary of the system is perceived as limited by the extent of hardware and software and direct human use and interaction. Second order IS (IS2), however, reflects Langefors' understanding (above) and includes a (socio-) technical system, together with other, further inter-human communication within an organisation or other human activity systems in social contexts - communities, groups etc. Such purposeful, human activity systems (Checkland, 1981; Langefors, 1995), are delimited by perceived boundaries of a social network, out of which a human activity system is perceived to emerge. We note that a 'system' is not to be considered as something having an 
objective existence (see for example Checkland, 1999) but rather as a mental construct which is created by an observer, who perceives system boundaries from her or his individual perspectives as meaningful for particular purposes. While the IS1 conception is useful when considering technical issues, analysis and design of effective business processes clearly requires a mind-set compatible with IS2, and an open-systems, socio-technical approach.

Process mapping to support effective flows of work and design of associated work systems requires a tool-set to support and guide participants in co-creating understandings of multiple levels of inherent contextual dependencies. Socio-technical tools and techniques are available that enable participants to explore their work situations and gradually co-create a rich pool of 'knowledge' as a basis for informed decisions (Bednar, et al, 2014). Ownership of this ongoing process must rest with the actors themselves, with help and guidance from experienced facilitators. Context is directly influenced by the presence and activities of engaged teams, as was evident in the GOSH we have discussed.

Bateson (1979, p.99) defines information as an instance where a person notices 'a difference that makes a difference' when engaging in sense-making practice. In seeking to inform themselves about the context of a systems development, users and their professional advisors need to consider not just what is commonly held to be 'best practice' or seek for a general consensus, but to consider the divergence of opinion including all participants' viewpoints. In such situations of complex knowledge sharing, a model for non-competitive benchmarking (such as the one illustrated in the GOSH example) makes sense. Similar approaches have been suggested elsewhere, e.g. Lego ${ }^{\circledR}$ have developed a 'serious play' toolkit which can be used by groups of individuals to explore a complex problem space by co-creating and sharing embodied narratives, which has been used successfully in supporting business development (see Kristiansen and Rasmussen, 2014). The explanatory materials for this kit suggest:

It is very important that each participant gets the chance to share the story about their model. The sharing is in itself a reflection process, in that when they share their models, participants explore their own expressions more closely. Those listening also have an opportunity to explore in more detail what the narrator expresses through the model.' (Open Source, 2011, n.p.).

This appears to reflect an open systems approach to surfacing and sharing tacit knowledge. Many other toolsets have emerged from practice in particular professions and trades to achieve a similar purpose, e.g. an architect wishing to discuss design choices with clients will use scaled-down, 3-D models suggestive of the actual dimensions, layout and appearance of the imagined building.

It is recognized that professionals are often constrained to work within a first order ISinfluenced interpretation of their roles. Furthermore the emphasis in their work is often placed on efficiency and productivity rather than effectiveness when determining what people 'need'. We have problematized elsewhere the idea that a professional developer can rely on 
technical expertise to produce a useful system on behalf of 'users' (Bednar and Welch, 2009). The authors argue that, too often, change agents and analysts try to act on behalf of engaged stakeholders in order to 'give them what they need' and are unaware of the opportunities provided by resources inside the communities they target. The insider knowledge within organizational communities and associated sense-making experience may be unknown to the analyst or may not be viewed as an asset. Thus it is not enough for analysts and developers to use an interventionist approach that focuses on situations without regard for the individuals involved.

In pursuit of excellence, developers need to support contextual inquiry into motivation data (what people want), and an analysis compatible with a second order IS interpretation. While we have demonstrated that non-competitive benchmarking, as practiced by GOSH, can be a very effective tool in process design, it can be expensive in practice and is not one that is open to all designers in respect of all processes. It is therefore appropriate to consider whether it is possible to share generalised ideas of 'good practice' without falling into a trap of emulating practices that may be appropriate in one context but not readily translated to another without reflection. We suggest that a tool-set making use of appropriate templates may be one way forward. Templates can be created that prompt engaged actors to think about critical issues for design, identified through experience of practice in a range of settings, without constraining the results of their individual and collecting thinking about contextual dependencies. These can be used in conjunction with collaborative, exploratory sociotechnical techniques such as mind-mapping, construction of rich pictures (Bednar and Day, 2009) and use of Lego® serious play, as well as boundary questions (Ulrich, 1994). As well as support for detailed analysis and design of processes, templates are available to highlight underpinning principles, such as readiness for change, sustainability (see e.g. Willard, 2012) and realisation of benefits from proposed changes (Ward and Daniel, 2012; Bednar and Welch, 2013).

An example of a socio-technical toolbox (Bednar, et al, 2014), which supports an open systems approach to analysis and design of work systems, uses the ETHICS methodology of Mumford (2003) as a frame of reference. This toolbox consists of over 20 different templates for analysis, each containing advice about its use in practice. The objective for each template is to support creation of analysis, reflection and design. In design of work and technical support systems, problem analysis, problem space identification and contextualization lead to deepened understandings of contextual dependencies and creation of possible resolutions. The documentation incorporates advice to guide discussion of wishes, possibilities and strategies, none of which is 'correct' but any of which can contribute towards a co-created resolution. Like non-competitve benchmarking, this combines analytical and convergent thinking with discursive approaches supporting divergent thinking (Bednar, et al, 2014).

We believe that the case of Great Ormond Street Hospital discussed above fits comfortably within the tradition, experiences and evolution of socio-technical approaches and practices, confirming that socio-technical approaches are still very relevant in 21 st century contexts. 
We also put forward a suggestion that reflection upon issues in best practice through appropriate toolsets can support collaborative inquiry into socio-technical design. We advocate wider use of open systems approaches such as these in efforts to create systems which will be experienced as useful in analysis and design practice.

\section{References}

Ahmed, P.K. and Rafiq, M. (1998). 'Integrated benchmarking: a holistic examination of selected techniques for benchmarking analysis', Benchmarking for Quality management \& Technology, 5(3) 225-242

Bateson, G. (1972). Steps to an Ecology of Mind. Chicago University Press.

Bateson, G. (1979). Mind and nature: A necessary unity. New York: Dutton.

Bednar, P.M. (2000). A Contextual Integration of Individual and Organizational Learning Perspectives as Part of IS Analysis, Informing Science: Journal of an Emerging Transdiscipline, 3(3), 145 - 156.

Bednar, P.M. (2007). Individual Emergence in Contextual Analysis, Problems of Individual Emergence, Special issue of Systemica, 14(1-6), 23-38.

Bednar, P.M and Day, L. (2009). 'Systemic combinatory use of Brainstorming, Mind-Maps and Rich Pictures for analysis of complex problem spaces'. Proceedings of the 8th European Conference on Research Methods in Business and Management, Valetta, Malta, 22-23 June 2009, 38-47.

Bednar, P.M., Sadok, M. and Shidarova, V. (2014). Socio-Technical Toolbox for Business Analysis in Practice, in Caporarello, Leonardo., Di Martino, Beniamino and Martinez, Marcello (Eds.). Smart Organizations and Smart Artifacts: Fostering Interaction Between People, Technologies and Processes. Lecture Notes in Information Systems and Organisation, Vol. 7. pp 219-228. Springer: Berlin, Heidelberg.

Bednar, P. and Welch, C. (2006). 'Structuring uncertainty: sponsoring innovation and creativity', in Creativity and Innovation in Decision Making and Decision Support, Vol.2. F. Adam et al, (editors). Decision Support Press.

Bednar, P.M. and Welch, C. (2009). Information Technology Projects - leaving the 'magic' to the 'wizards' in Papadopoulos, G. A., et al. (eds) (2008), Information Systems Development: Towards a Service Provision Society, New York: Springer-Verlag.

Bednar, P.M. and Welch, C. (2009). Contextual Inquiry and Requirements Shaping, in C. Barry, K. Con-boy, M. Lang, G. Wojtkowski, and W. Wojtkowski (editors), Information Systems Development: Challenges in Practice, Theory, and Education (Vol.1), 225-236

Bednar, P.M. and Welch, C. (2013). A Case for Multi Criteria Benefit Analysis. Organizational Change and Information Systems, Lecture Notes in Information Systems and Organisation, Vol. 2, pp 337-344.

Boxwell, R.J. (1994). Benchmarking for Competitive Advantage, New York: McGraw-Hill

Catchpole, K; De Leval, M; McEwan, A; Pigott, N; Elliott, M; McQuilan, A; MacDonauld, C; Goldman, A (2007). Patient Handover from Surgery to Intensive Care: Using Formula 1 
and Aviation Models to Improve Safety and Quality. Paediatric Anaesthesia, 17(5) 470478.

Checkland, P 1999, Soft Systems Methodology in Action. John Wiley and Sons Ltd, Chichester.

De Leval, M., Carthy, J. Wright, D., et al (2000). Human factors and cardiac surgery. Journal of Thoracic Cardiovascular Surgery, 119, 661-672.

Dorsch, J. and Yasin, M. (1998). 'A framework for benchmarking in the public sector,' International Journal of Public Sector Management, 11(2/3), 91-115

Elmuti, D. and Kathawala, Y. (1997). 'An overview of benchmarking processes', Benchmarking for Quality management \& Technology, 4(4), 229-243.

Kristiansen, P. and Rasmussen, R. (2014). Building a Better Business Using the Lego Serious Play Method. Chichester: John Wiley \& Sons

Moon, Y. B. (2007). Enterprise resource planning (ERP): A review of the literature. International Journal of Management and Enterprise Development, 4(3): 235-263.

Mumford, E. (2003). Redesigning Human Systems. Cambridge: IRM Press.

Mumford, E. (2006). The story of socio-technical design: reflections in its successes, failures and potential. Information Systems Journal, 16(4), 317-342.

Open-source/Introduction to Lego®SeriousPlay, accessed 13 June 2011 https://dl.dropbox.com/u/5032997/LEGO\%20Serious\%20Play\%20OS/LEGO\%C2\%AE_S ERIOUS_PLAY_OpenSource.pdf

Schein, E (1992), Organizational Culture \& Leadership, $2^{\text {nd }}$ edition, San Francisco: JosseyBass

Slack, N., Chambers, S. and Johnston, R. (2010). Operations Management. Cambridge: Pearson

Sower, V., Duffy, J.A. and Kohers, G. (2008). Ferrari's Formula One Handovers and Handovers from Surgery to Intensive Care. Chapter 10 in Benchmarking for Hospitals: achieving best-in-class performance without having to reinvent the wheel. ASQ Press.

Ulrich, W. (1994). Critical heuristics of social planning: A new approach to practical philosophy, 2nd edition, New York and London: John Wiley \& Sons.

Ward, J. and Daniel, E. (2012). Benefits Management: How to Increase the Business Value of Your IT Projects. $2^{\text {nd }}$ edition, Chichester: John Wiley \& Sons.

Weick, K.E. and Sutcliffe, K.M. (2007). Managing the Unexpected: Resilient Performance in an Age of Uncertainty. $2^{\text {nd }}$ Edition, Chichester: John Wiley \& Sons.

Willard, B. (2012). New Sustainability Advantage: Seven Business Case Benefits from the Triple Bottom Line. Gabriola Island, BC: New Society Publishers; Tenth Anniversary Edition.

Yasar, F. and Zairi, M. (2010). 'Best practice transfer for future competitiveness,' Total Quality Management \& Business Excellence, 11(4), 734-740 